\title{
Business intelligence como ferramenta gerencial no suporte ao processo de business performance management
}

\author{
Alvaro Vieira Lima \\ Davi Marques Lima²
}

\section{Resumo}

O objetivo deste trabalho é desenvolver o conceito de Business Intelligence aplicado ao processo de Business Performance Management, utilizando uma abordagem fenomenológica de pesquisa. Partindo do desenvolvimento dos conceitos apresentados em diversos artigos e livros, aliado ao estudo de três casos selecionados, pretende-se caracterizar a utilização do referido instrumento como um valioso suporte ao processo de gestão empresarial com foco em medidas de resultado.

Palavras-chave: Business intelligence. Medidas de desempenho. Controle gerencial.

\section{Introdução}

No atual cenário de negócios, a diferenciação e a busca por um desempenho de destaque, seja a empresa de que ramo for, dar-se-ão por meio da utilização de recursos que visam à execução ótima das atividades, em paralelo à redução de seus custos.

Por intermédio dos elementos da tecnologia de informação, é possível armazenar e acessar de maneira eficaz e estruturada, informações sobre fornecedores, clientes e suas tendências de consumo, concorrentes e demais elementos de gestão da própria empresa, além de viabilizar a interlocução dos participantes, bem como a análise dos dados obtidos, no sentido de operar de maneira segura e objetiva.

\footnotetext{
1 Professor Adjunto do Departamento de Ciências Contábeis da Faculdade de Administração e Finanças da UERJ - Universidade do Estado do Rio de Janeiro.

2 Bacharel em Sistemas de Informação pela PUC-RJ - Pontifícia Universidade Católica do Rio de Janeiro, Analista de sistemas da FINEP - Financiadora de Estudos e Projetos .
} 
Na lógica contemporânea dos negócios, compreender como as informações permeiam os níveis estratégico, tático e operacional das organizações é fundamental para o seu sucesso. As diversas características das informações, tais como, fonte, grau de repetição, precisão, assim como as diversas formas de classificá-las, são determinantes na escolha das ferramentas que serão utilizadas no seu tratamento. Só assim, é possível viabilizar o processo de tomada de decisões rápidas e eficazes, de modo a ombrear a organização com seus competidores e tentar batê-los.

Business Intelligence é o recurso do qual as tecnologias de informação lançam mão para desafiar o volume de dados multiplicados a todo tempo no ambiente empresarial. Os desafios enfrentados pelas empresas viabilizam a produção de tecnologias que se prontifiquem a atendê-las, e é disso que o presente artigo vem tratar. Pretende-se discorrer, de maneira objetiva e clara, sobre as estratégias de aplicação, benefícios e possibilidades de ganhos reais que se podem extrair desse recurso.

Analisar-se-á em três empresas de diferentes ramos as questões que se colocam em face da necessidade de integração de dados: os problemas que se apresentam, as soluções de tecnologia de informação que se podem pensar para elas, e os resultados e benefícios, principalmente nas decisões gerenciais que podem emergir a partir de sua implementação.

\section{Revisão da literatura}

\subsection{Business intelligence}

\subsubsection{Definição}

Segundo Turban (2008, p. 27), Business Intelligence (BI) "é um termo guarda-chuva que inclui arquiteturas, ferramentas, bancos de dados, aplicações e metodologias". Entretanto, em virtude de ser uma expressão livre de conteúdo, pode transmitir significados diversos, gerando certa confusão em torno desse conceito. 
Ainda na visão de Turban (2008, p. 32), os principais objetivos do BI são: "permitir o acesso interativo aos dados (às vezes, em tempo real), proporcionar a manipulação desses dados e fornecer aos gerentes e analistas de negócios a capacidade de realizar a análise adequada". Ao utilizarem uma boa ferramenta de BI, os executivos e gerentes podem analisar situações e desempenhos já enfrentados, os quais servirão de base ao refinamento e aprimoramento das atuais decisões. Turban (2008, p. 33) resumem o processo de BI como "a transformação de dados em informações, depois em decisões e finalmente em ações”.

\subsubsection{Histórico}

O termo BI foi criado na década de 1990 pelo Gartner Group. Porém, segundo Turban (2008, p. 27), "o conceito se iniciou muito antes, com suas raízes nos sistemas de geração de relatórios SIG - Sistemas de Informações Gerenciais - (do inglês, Management Information Systems - MIS) dos anos 1970". Todavia, esses sistemas tinham seu foco na geração de relatórios estáticos e bidimensionais, limitados pelos recursos disponíveis àquela época.

Com a evolução da Tecnologia da Informação, surgiu, no início dos anos 1980, o conceito de sistemas de informação executiva (do inglês, Executive Information Systems - EIS), elevando o suporte de sistemas automatizados do nível operacional para os níveis tático e estratégico. Esse conceito introduziu sistemas de geração de relatórios dinâmicos multidimensionais, prognósticos e previsões, análises de tendências, possibilidades de maior detalhamento, acesso a status e fatores críticos de sucesso.

Segundo Turban (2008, p. 28), “esses recursos apareceram em dezenas de produtos comerciais até o meio da década de 1990. Depois, os mesmos recursos e alguns recursos novos apareceram sob o nome de BI". Turban et al (2008, p. 28) ainda acrescentam que hoje "se reconhece que todas as informações de que os executivos necessitam podem estar em um bom sistema de informações baseado em BI. Assim, o conceito original de Sistemas de Informações Executivas foi transformado em BI". 


\subsubsection{Arquitetura e componentes}

O BI tem quatro grandes componentes: um data warehouse (DW) com seus dados-fonte; a análise de negócios, uma coleção de ferramentas para manipular e analisar os dados no data warehouse, incluindo data mining; Business Performance Management (BPM) para monitoria e análise do desempenho; e uma interface de usuário (como o dashboard) (TURBAN et al, 2008). Esses quatro componentes são analisados mais detalhadamente a seguir:

Data warehouse é um banco de dados estruturado para oferecer suporte à tomada de decisões gerenciais. Esse tipo de banco de dados contém uma grande variedade de elementos que ensejam a construção de uma imagem coerente das condições da organização em um determinado período no tempo. A ideia principal de um DW é fornecer uma infraestrutura de banco de dados que esteja sempre online e contenha todas as informações dos sistemas operacionais da empresa, incluindo dados históricos;

As ferramentas são quaisquer tipos de software que permitam ao usuário criar relatórios e consultas sob demanda, além de realizar análises de dados. Essas ferramentas surgiram originalmente com o nome de processamento analítico online (do inglês, Online Analytical Processing - OLAP);

Business Performance Management, segundo Turban et al (2008), é considerado o componente final do processo de Business Intelligence. Este utiliza a análise, a geração de relatórios e as consultas de BI com o objetivo de aperfeiçoar o desempenho geral da organização; e

Interfaces de usuários são as ferramentas de visualização que apresentam as informações de uma maneira compreensível aos usuários. Estas podem ser dashboards (fornecem uma visão abrangente e amigável dos indicadores chaves de desempenho e suas tendências e exceções), cubo multidimensional de dados e, até mesmo, realidade virtual em alguns casos.

\subsubsection{Benefícios}

Eckerson (2003) relata, a partir dos resultados de uma pesquisa entre 510 empresas, que os benefícios do BI são: 
Economia de tempo (61\%);

Versão única da verdade (59\%);

Melhores estratégias e planos (57\%);

Melhores decisões táticas (56\%);

Processos mais eficientes (55\%); e

Economia de custos (37\%).

Em outra pesquisa, Thompson (2004) revela que os maiores benefícios do BI são:

Geração de relatórios mais rápida e precisa (81\%);

Melhor tomada de decisões (78\%);

Melhor serviço ao cliente (56\%); e

Maior receita (49\%).

Entretanto, Turban (2008) afirmam que mensurar o valor para o negócio do BI é ainda difícil, pois as metodologias para avaliá-lo são complexas devido aos muitos benefícios intangíveis e ao fato do data warehouse ser uma infraestrutura que serve também para muitas outras aplicações da empresa.

\subsubsection{Presente e futuro}

É um consenso geral que no atual ambiente de negócios altamente competitivo, a qualidade e a rapidez na troca de informações não são mais uma escolha, mas uma questão de sobrevivência. Portanto, é de suma importância levar em consideração os benefícios do Business Intelligence. Segundo Baum (2006, p. 1), "relatórios recentes de analistas do setor mostraram que, nos próximos anos, milhões de pessoas usarão ferramentas visuais de BI e análise de BI todos os dias".

As organizações que se utilizam de BI estão obtendo mais valor por meio do acesso a múltiplas informações por todos os níveis da organização, maximizando o uso de seus ativos, representados, nesse caso, por informações valiosas (TURBAN, 2008).

As ferramentas de BI não estão mais somente nas mãos das grandes empresas como há alguns anos atrás. Hoje, produtores, varejistas, governos e demais 
agentes do mercado já utilizam essa ferramenta. Tudo indica que esses instrumentos irão se generalizar em todas as atividades, oferecendo praticamente qualquer tipo de análise e facilitando cada vez mais o processo decisório em todos os níveis.

Segundo Turban (2008, p. 47),

[...] o business intelligence está abrindo suas asas para abraçar todos, desde pequenas e médias empresas até grandes organizações. Ferramentas de análise para funções muito específicas também estão entrando no mercado, e elas ajudarão algumas empresas a adotar apenas a análise em vez da implementação completa de BI baseada em data warehouse.

\subsection{Business performance management}

\subsubsection{Definição}

Segundo Coveney (2003), BPM tem uma abordagem holística para a implementação e acompanhamento da estratégia. Ele combina metodologias de negócios, tais como scorecards, valor econômico adicionado (do inglês, Economic Value Added - EVA) e gestão baseada em atividades; métricas, que são as medidas específicas utilizadas nessas metodologias; processos, que são os procedimentos que uma organização segue para implementar e monitorar o desempenho de seus negócios; e sistemas, que são as soluções tecnológicas que combinam as metodologias, métricas e processos de uma única empresa ao nível de sistema gestão. Além de ser constituído por uma única aplicação, um sistema BPM difere das outras abordagens para a gestão de desempenho em razão de utilizar a tecnologia aliada às melhores práticas empresariais, a fim de ajudar executivos a responderem a questões fundamentais em torno da formulação e implementação da estratégia (COVENEY, 2003).

\subsubsection{Histórico}

Segundo Coveney (2003), as três últimas décadas foram marcadas por uma série de inovações que transformaram a forma como as organizações planejam 
e acompanham seu desempenho. Na década de 1970, os Sistemas de Suporte a Decisão (do inglês, Decision Suport Systems - DSS) serviram de base para as organizações modelarem seu futuro. Usando tecnologias multidimensionais baseadas em mainframes, gerentes financeiros e gerentes operacionais podiam analisar e planejar suas ações por canal de distribuição, cliente, linha de produtos, e mais, dando-lhes a capacidade de localizar e explorar oportunidades de mercado.

Na década de 1980, os Sistemas de Informação Executiva (do inglês, Executive Information Systems - EIS), ofereceram aos CEO's e demais executivos a tecnologia que estes poderiam utilizar para investigar os pontos fortes e fracos de sua organização, sem ter que obter a assistência de programadores. Com o tempo, esses sistemas se estenderam aos demais níveis de gestão e passaram a ser utilizado em toda a organização.

Na década de 1990, o ritmo dos negócios se acelerou dramaticamente. O Business Intelligence (BI) tornou-se a chave para acompanhar os processos de planejamento, análise e elaboração de relatórios. Os Sistemas Transacionais (sistemas comuns de armazenamento de dados) ficaram sob os holofotes, já que as organizações necessitavam torná-los mais eficientes. Sistemas de Enterprise Resource Planning (ERP) tornaram-se uma ferramenta imprescindível a toda empresa.

Ao mesmo tempo, a crescente disponibilidade de computadores e conexões em tempo real resultou na proliferação de sistemas de usuários finais, os quais podiam analisar os dados assim que se tornavam disponíveis. Soluções especializadas, tais como sistemas de gestão das relações com clientes (do inglês, Customer Relantionship Management - CRM), também explodiram na área de negócios.

Ainda de acordo com Coveney (2003), muito embora essas inovações estivessem acontecendo, ainda havia uma crescente sensação de que a tecnologia por si só não era a resposta. Apesar de estes desenvolvimentos terem produzido como resultado a disponibilização de um maior volume de informações rapidamente acessível, nenhum deles foi particularmente útil para os gestores que lutavam por encontrar maneiras de permitir uma melhor ação estratégica. 
Durante a década de 1990, muitos livros e artigos foram escritos sobre metodologias de gestão que ensejassem a melhor forma de implementar estratégias, dentre eles o Balanced Scorecard (BSC), possivelmente o mais conhecido. O BSC enfatizou que as organizações devem planejar e monitorar todos os aspectos do negócio e não apenas os resultados financeiros. Isso incluía retenção de clientes, rotinas de trabalho, eficiência interna, aprendizado e crescimento. Mas, embora essas metodologias fossem boas em si mesmas, não ofereceram respostas a todos os desafios gerenciais.

Apenas quando são combinadas com diferentes metodologias de gestão de processos (como, por exemplo, orçamento, previsão de atividades, relatórios de gestão), para programar e medir os elementos adequadamente apoiados pela tecnologia, é que as organizações podem realmente começar a gerir a execução da estratégia. E isso é o Business Performance Management (COVENEY, 2003).

\subsubsection{Metodologias}

Segundo Turban (2008), as metodologias mais utilizadas em um sistema BPM são:

Balanced Scorecard (BSC): provavelmente, o sistema de gerenciamento de desempenho mais conhecido e amplamente utilizado. Nos últimos anos, o BSC se tornou um termo genérico que é utilizado para representar quase todo o tipo de aplicação e implementação de painel. De um ponto de vista mais abrangente, o BSC é uma medida de desempenho tanto quanto uma metodologia de gerenciamento, que ajuda a traduzir os objetivos e metas financeiras de clientes, de processos internos e de aprendizado e de crescimento de uma empresa em um conjunto de iniciativas mensuráveis, como uma metodologia de medida; o BSC é planejado para superar as limitações de sistemas que têm foco financeiro. Ele faz isso, traduzindo a visão e estratégia de uma empresa em um conjunto de objetivos financeiros, não-financeiros, medidas, metas e iniciativas (TURBAN, 2008); 
Six Sigma (Seis Sigmas): é um conjunto de práticas originalmente desenvolvidas pela Motorola para melhorar sistematicamente os processos ao eliminar defeitos. Um defeito é definido como a não conformidade de um produto ou serviço com suas especificações. Seis Sigmas também é definido como uma estratégia gerencial para promover mudanças nas organizações, fazendo com que se chegue a melhorias nos processos, produtos e serviços para a satisfação dos clientes. Diferente de outras formas de gerenciamento de processos produtivos ou administrativos, o Seis Sigmas tem como prioridade a obtenção de resultados de forma planejada e clara tanto de qualidade como, sobretudo, de financeiros. A qualidade não é vista pelo Seis Sigmas na sua forma tradicional, isto é, a simples conformidade com normas e requisitos da organização. Ele a define como o valor agregado por um amplo esforço de produção com a finalidade de atingir objetivos definidos na estratégia organizacional (CARVALHO et al., 2006).

\section{Metodologia}

\subsection{Classificação da pesquisa}

A presente pesquisa classifica-se, quanto aos fins, como sendo qualitativa, descritiva e aplicada, e, quanto aos meios, como documental, bibliográfica e estudo de caso.

Com esta pesquisa, buscou-se analisar e ressaltar a importância da utilização do Business Intelligence no gerenciamento estratégico de uma empresa por meio da coleta e análise de dados relevantes, tais como os casos exemplificados durante o trabalho.

Na fase de levantamento de dados, executou-se uma pesquisa bibliográfica por intermédio do levantamento dos trabalhos realizados e relevantes com o tema desta pesquisa, assim como a análise de casos de sucesso da implementação de ferramentas de Business Intelligence. 


\subsection{Premissas}

As premissas básicas formuladas a serem avaliadas no presente trabalho são:

- A utilização de uma ferramenta de Business Intelligence melhora a qualidade no processo de gerenciamento estratégico;

- Uma ferramenta de Business Intelligence pode ser considerada uma ferramenta de controle gerencial; e

- O Business Intelligence é uma ferramenta de auxílio ao Business Performance Management.

\subsection{Coleta e análise de dados}

Os dados coletados foram do tipo secundário e foram coletados por meio de levantamento bibliográfico, sites da web e de outros trabalhos acadêmicos correlatos, assim como levantamento de estudos de casos de sucesso da utilização de BI disponíveis na Internet, com o objetivo de embasar o conhecimento do assunto tratado.

As empresas cujos casos foram estudados são:

- Quelle BmbH (Principal sede em: Furth, Alemanha).

- China Yangtze Power Co., Ltd. (Principal sede em: Pequim, China).

- Toyota Motor Sales U.S.A. (Principal sede em: Torrance, California, EUA).

Todas as informações foram obtidas da revista Business Benefit Series (2010) produzida pela Oracle. 


\section{Resultados e discussão}

\subsection{Quelle GmbH}

\subsubsection{Empresa}

A Quelle GmbH é uma empresa europeia que atua no segmento de correspondências e varejo. Atua em 15 países e possui uma rede de 4000 lojas varejistas, com a opção de compra pela Internet e pelo telefone. Fornecedora multifacetada e parceira da empresa alemã Arcandor AG, pretende realizar expansões pelo centro e nordeste da Europa e Rússia.

\subsubsection{Problema}

Por motivos de ordem histórica, a Quelle $G m b H$ vem armazenando os dados e informações acerca dos clientes (comportamentos, informações sobre poder aquisitivo, informações sociodemográficas) em diferentes bancos de dados, gerados em diferentes momentos e com diferentes objetivos. O desafio seria tornar disponível todos esses dados de forma cumulativa. Integração e acessibilidade eram questões precárias no sistema de dados antes utilizado, e a pretensão era implantar novos sistemas que, concomitantemente, reduzissem os custos no armazenamento de informações e melhorassem o desempenho.

Uma nova gestão de sistema de informações se fazia necessária no sentido de não só estar voltada para o desenvolvimento empresaria, como também de facilitar o trânsito de informações irrestritas e completas sobre o cliente. A coleta e o armazenamento de dados mostravam-se insatisfatórias para a produção de análises estratégicas consistentes acerca do cliente e suas demandas; era preciso alinhá-los e estudá-los.

\subsubsection{Solução}

A partir da substituição de uma arquitetura simétrica de multiprocessamento por um sistema Oracle Real Application Clusters, atingiu-se um maior de- 
sempenho no sentido de integrar diversas fontes de dados e desenvolvimento de uma plataforma eficiente e inteiramente disponível, contendo a totalidade dos bancos de dados da empresa. $\mathrm{O}$ armazenamento de dados era, agora, uma potente ferramenta para mediar, não só o gerenciamento da relação com os clientes, como também um poderoso indicador para a tomada de decisões estratégicas.

A integração de 12 servidores, atingindo a capacidade de 17 terabytes, duplicou o desempenho e reduziu os custos à metade. Além disso, a dificuldade de manuseio e utilização do sistema pelos usuários foi minimizada de forma que se tornou possível gerar relatórios com mudanças de parâmetros e formas sem o auxílio da equipe de TI.

A possibilidade de gerar conhecimento consistente sobre o cliente, por meio do atrelamento de informações e da confrontação de dados, produziu uma relação de eficácia na produção de campanhas e na avaliação do efeito delas para cada público-alvo. O sistema autoriza ainda a criação de perfis para os clientes, facilitando o agrupamento por características, e a previsibilidade de comportamentos futuros dos clientes, tais como futura adesão de produtos, expectativa de certos comportamentos etc.

\subsubsection{Benefícios após BI}

- Redução de 30\% dos custos por meio da nova infraestrutura de TI implantada;

- Integração de variadas fontes de dados;

- Criação de um gestor de informações com 300 indicadores para 10 dimensões;

- Maior assertividade na análise de dados relativos à gestão do relacionamento com o cliente; $\mathrm{e}$

- Aumento na eficácia das campanhas de marketing. 


\subsection{China yangtze power co. Itd.}

\subsubsection{Empresa}

Yangtze Power Co. Ltd é a maior empresa de energia listada publicamente na China. O Three Gorges Project Corporation, desde 2002, supervisiona usinas hidroelétricas criadas como parte do projeto Three Gorges Dam. Quando concluído, em 2011, a barragem será capaz de gerar 22.500 megawatts de eletricidade, tornando-se a maior estação de energia hidroelétrica do mundo por capacidade. A Yangzte Power, atualmente, gera a Gezhouba Power Station e seis unidades geradoras comissionadas.

\subsubsection{Problema}

A Yangzte Power é uma empresa que se utiliza eminentemente de tecnologia para gerir-se, de modo que, cada departamento da empresa possuía um sistema e base de dados próprios, cada qual operando separadamente, sendo necessário, muitas vezes, que os funcionários solicitassem aos de outros departamentos informações via e-mail ou telefone. Dessa forma, as análises eram então produzidas numa lógica departamentalizada, gerando relatórios sem possibilidade de interlocução.

No sentido de atingir uma corporeidade empresarial e obter uma visão coerente de seu funcionamento, a empresa coletava os dados desses sistemas fragmentados e os reunia com a finalidade de confrontá-los, relacioná-los e estudá-los, visando à análise do desempenho total da empresa. Tal procedimento constituía-se numa operação longa e laboriosa, que precarizava a possibilidade de chegar-se a conclusões imediatas e claras sobre os impactos das ações empregadas e dos resultados das estratégias adotadas pela própria empresa.

\subsubsection{Solução}

Na esteira de uma expansão empresarial, a Yangzte Power embarcou em projetos de desenvolvimento de sistemas, infraestrutura e práticas de gerencia- 
mento. Introduziu, em 2007, um sistema de banco de dados Oracle, bem como suas ferramentas de Business Intelligence.

O novo recurso implantado viabilizaria a integração de seus sistemas em um único, em que todos os dados estivessem acessíveis a quaisquer departamentos da empresa, produzindo uma unificação corporativa, um alcance incomparável das estimativas e a própria possibilidade de produção de questões a partir dos resultados.

O novo sistema podia, ainda, produzir relatórios, gráficos e planilhas adaptáveis às informações que se desejasse relacionar e comparar, bem como gerar instantaneamente uma descrição minuciosa de todas as atividades da empresa, introduzindo a possibilidade de antevisão dos efeitos e impactos das decisões estratégicas tomadas pela empresa e da miríade de questões antes desconhecidas ao grupo gerencial da empresa.

\subsubsection{Benefícios após BI}

- Transição de 10 fontes de informações diferentes para um único sistema de informações integrado;

- Possibilidade de acessar os dados disponibilizados no dia anterior às 9 horas da manhã do próximo dia;

- Possibilidade para os usuários de gerar relatórios e gráficos adaptados às suas necessidades específicas;

- Tomada de decisões estratégicas a partir de análises devidamente abalizadas; e

- Gerenciamento com ampla visão empresarial de seu funcionamento microscópico. 


\subsection{Toyota motor sales U.S.A.}

\subsubsection{Empresa}

A Toyota Motor Sales (TMS) foi fundada há aproximadamente 50 anos, assim como a divisão de vendas, marketing e distribuição da Toyota Motor Corporation (TMC). A TMS abriu suas portas para os negócios em 1957, em Holywood, Califórnia, vendendo 287 carros Toyopet (um dos primeiros modelos da Toyota) e um Land Cruiser, naquele primeiro ano. Quase meio século depois, a TMS se transformou em uma indústria automotiva americana bilionária, dominando o mercado americano de carros e conquistando grande credibilidade com qualidade e confiabilidade. Embora a Toyota e a Lexus sejam grandes marcas, a TMS experimentou um forte crescimento nos últimos anos com novas entradas no mercado, como o carro Prius e a linha de carros Scion, voltada para o publico jovem.

\subsubsection{Problema}

Embora o crescimento fenomenal da Toyota nos EUA fosse indiscutível, era preciso garantir o crescimento de maneira sustentável e lucrativa. Tracey Doi, vice-presidente e diretora financeira do grupo, deu-se conta que era preciso mais do que apenas coletar dados e relatórios financeiros para enviar à matriz no Japão; fazia-se mister debruçar-se, cuidadosamente, sobre sua unidade empresarial.

A geração de dados de ordem contábil e financeira era enormemente volumosa, despendendo grande tempo da equipe financeira para o processamento dos mesmos, sobrando pouco tempo para obtenção de uma análise voltada para o crescimento da empresa a partir do feedback dos dados estudados.

A política de desenvolvimento empresarial, voltada para a criação de valor de mercado, acabava por ser negligenciada, uma vez que os executivos tinham a maior parte de seu tempo tomada por um processamento que poderia ser realizado de outra forma, incluindo a possibilidade de migração para um ambiente terceirizado ou minimamente compartilhado. 


\subsubsection{Solução}

Simplificar os procedimentos financeiros, produzir um alinhamento junto a seus clientes e prover seus gerentes com as informações apropriadas para dar suporte aos objetivos de crescimento da empresa eram os principais pontos propostos por Doi para alavancar o desempenho e ensejar o surgimento de pessoas com valores voltados para pensar a própria empresa e suas questões.

Para tanto, utilizou-se o software Peoplesoft bem como o sistema Kaizen, aliando o armazenamento inteligente para todos os tipos de dados, conteúdo corporativo e serviços em um meio unificado, personalizado e seguro, gestão do crescimento do volume de informações com desempenho e confiabilidade, além de um gerenciamento de fatores, recursos humanos e banco de dados de clientes.

Por intermédio de meios sólidos e customizados para a coleta de dados relativos a clientes e mercado obtidos a partir dos softwares implantados, tornou-se possível a tomada de decisões de maneira mais acertada. Dessa forma, foi possível atingir um alto desempenho e uma diferenciação mercadológica obtida pelo progresso alcançado pelos métodos instituídos.

\subsubsection{Benefícios após BI}

- Infraestrutura virtual, ganhos reais;

- Padronização dos processos de gestão;

- Contínua redução de custos, por meio, por exemplo, da adesão de cartões de débito; e

- Tomada de decisões estratégicas baseadas em dados seguros e acessíveis.

\section{Conclusão}

Ao traçar um paralelo entre os problemas e as soluções encontradas nas três empresas abordadas neste trabalho, pode-se perceber que a maior dificuldade co- 
mum entre elas era alinhar o uso da Tecnologia da Informação com seus negócios, sobretudo no que tange ao processo de tomada de decisões gerenciais nos diversos níveis das organizações.

Um dos principais problemas enfrentados foi o uso de Sistemas de Informações não integrados para gerar medidas e métricas de desempenho organizacional. Como esses sistemas operavam separadamente, era muito custoso integrar suas informações. Os relatórios estratégicos, gerados a partir de dados colhidos de diversas fontes, usando tecnologias que não interagiam, eram precários, visto que os sistemas de informações implantados atuavam somente nas áreas operacional e tática dessas organizações. Como resultado, produziam-se tomadas de decisões, muitas vezes, ineficazes e ineficientes.

Como solução, foi empregado o conceito de Business Intelligence utilizando Sistemas Integrados de Gestão, com auxílio de datawarehouses (para armazenamento integrado e de larga escala de dados) e datamining (ferramenta para extrair informações). Essas implementações permitiram a criação de novas medidas e métricas para controlar o desempenho organizacional. Pôde-se perceber que o BPM dessas empresas estudadas foi aperfeiçoado, apresentando métricas e medidas de desempenho alinhadas com as características reais e de acordo com a estrutura organizacional das empresas.

O que se pode concluir a partir das ações implementadas nas três empresas de diferentes ramos é que a adoção dos recursos de Business Intelligence implicou, dentre outros, nos seguintes ganhos: enorme salto de desempenho; desaparecimento de fronteiras departamentais; integração de recursos; e a disponibilidade de dados de maneira eficiente, propiciando assim uma significante redução dos problemas anteriormente enfrentados.

Num ambiente que se globaliza e se modifica cada vez mais rapidamente, é preciso agregar ao conhecimento dos profissionais ferramentas que lhes deem a chance de, a partir de informações bem estruturadas, produzir análises mais rápidas, precisas e eficazes, ensejando decisões que contribuam para o crescimento sólido e sustentável das organizações. 
Cabe ressaltar que este trabalho baseou-se em dados secundários, utilizando três estudos de casos de sucesso da utilização de BI disponíveis na Internet. Seria de grande utilidade que outros estudos se valessem de pesquisas mais amplas que buscassem identificar dificuldades ou pontos negativos que podem surgir na implementação desse tipo de solução.

\section{Bussiness intelligence as a management tool supporting business performance management process}

\section{Abstract}

The present work aims to develop a Business Intelligence applied to the process of Business Performance Management concept, by using a phenomenological research approach. Based on the development of concepts presented in various articles and books along with the study of three selected cases, it intents to characterize the use of the mentioned instrument as a valuable support to business process management with a focus on outcome measures.

Keywords: Business Intelligence; Business Performance Management; Management Performance Control.

\section{Referências}

BAUM, D. The face of intelligence. ORACLE Magazine, mar. 2006. Disponível em: <http://www.oracle.com/technetwork/issue-archive/2006/06-mar/o26intelligence -100056.html>. Acesso em: 11 out. 2010.

CARVALHO, M. et al. Implementação de programas de qualidade: um survey em empresas de grande porte no Brasil. Revista Gestão \& Produção, 2006. Disponível em <http://www.scielo.br/pdf/gp/v13n2/31167.pdf>. Acesso em: 11 out. 2010. 
COVENEY, M. CPM: What it is and how it differs from traditional approaches? Disponível em: <http://www.businessforum.com/ Comshare01.html $>$. Acesso em: 11 out. 2010.

ECKERSON, W. Smart Companies in the 21 $1^{\text {st }}$ Century: the secrets of creating successful business intelligent solutions. Seattle, WA: The Data Warehousing Institute, 2003.

ELMASRI, R.; NAVATHE, S. Sistemas de banco de dados. 4. ed. São Paulo: A. Wesley, 2005.

MOTOROLA UNIVERSITY. What is six sigma? Disponível em: <http://www. motorola.com/content.jsp?globalObjectId=3088>. Acesso em: 11 out. 2010.

THOMPSON, O. Business intelligence success, lesson learned. Technology Evaluation, 2010. Disponível em: <http://www.sydmart.com/artic/bi_success. pdf >. Acesso em: 11 out. 2010.

TURBAN, E. Business intelligence: um enfoque gerencial para a inteligência do negócio. São Paulo: Bookman, 2009.

WARREN, C. Contabilidade gerencial. 2. ed. São Paulo: Thomson, 2008.

WILLIANS, S.; WILLIANS, N. The profit impact of business intelligence. San Francisco, CA: M. Kaufmann, 2007. 


\section{Para publicar na revista Universitas Gestão e TI, entre no endereço eletrônico www.publicacoesacademicas.uniceub.br. Observe as normas de publicação, facilitando e agilizando o trabalho de edição.}

